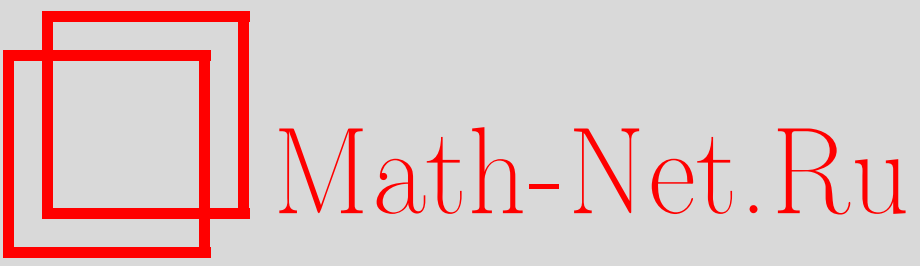

Л. А. Калякин, Асимптотики решений уравнений главного резонанса, ТМФ, 2003, том 137, номер 1, 142-152

DOI: https://doi.org/10.4213/tmf1757

Использование Общероссийского математического портала Math-Net.Ru подразумевает, что вы прочитали и согласны с пользовательским соглашением

http://www.mathnet.ru/rus/agreement

Параметры загрузки:

IP : 54.205 .225 .156

26 апреля 2023 г., 11:24:58

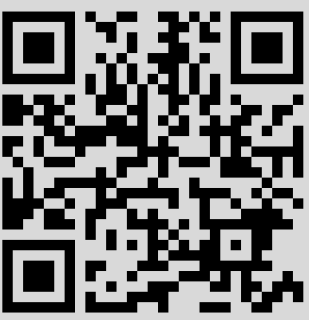


ТЕОРЕТИЧЕСКАЯ

И МАТЕМАТИЧЕСКАЯ

ФИЗИКА

Том 137, № 1

октябрь, 2003

(C) 2003 г.

Л. А. Калякин ${ }^{*}$

\section{АСИМПТОТИКИ РЕШЕНИЙ УРАВНЕНИЙ ГЛАВНОГО РЕЗОНАНСА}

Исследована система двух дифференциальных уравнений первого порядка, которая возникает при усреднении нелинейных систем по быстрым одночастотным колебаниям. Основной результат состоит в построении асимптотики двухпараметрического семейства решений с неограниченно растушей амплитудой. Кроме того, построена асимптотика другого двухпараметрического семейства решений с ограниченной амплитудой. Эти результаты, в частности, дают ключ к пониманию авторезонанса как явления значительного роста вынужденных нелинейных колебаний, инициированных малой внешней накачкой.

Ключевые слова: нелинейные уравнения, асимптотика, ВКБ-приближение.

\section{1. ПОСТАНОВКА ЗАДАЧИ}

Рассматривается система двух дифференциальных уравнений

$$
\frac{d}{d \theta}\left(\begin{array}{l}
x \\
y
\end{array}\right)+\left[\varphi(\theta)-\lambda\left(x^{2}+y^{2}\right)\right]\left(\begin{array}{c}
-y \\
x
\end{array}\right)=\left(\begin{array}{c}
f(\theta) \\
g(\theta)
\end{array}\right), \quad \lambda=\text { const } \neq 0,
$$

с частными случаями которой связано описание разного типа резонансных эффектов в нелинейных системах. Предполагается, что заданные функции $\varphi(\theta), f(\theta), g(\theta)$ являются гладкими (бесконечно дифференцируемыми при $\theta \geqslant 0$ ) и имеют степенное асимптотическое разложение при $\theta \rightarrow \infty$. При этом коэффициент $\varphi(\theta)$ линейно растет на бесконечности, а правые части ограничены и $f^{2}+g^{2} \rightarrow$ const $\neq 0$. Требуется построить асимптотику на бесконечности (при $\theta \rightarrow \infty$ ) для различных решений этой системы.

Имея в виду линейные преобразования переменных $x, y, \theta$, которые сохраняют структуру уравнений, можно считать без ограничения общности, что асимптотические разложения исходных функций имеют специфические главные члены ${ }^{1}$ )

$$
\varphi(\theta)=\theta+\sum_{n=1}^{\infty} \phi_{n} \theta^{-n}, \quad f(\theta)=1+\sum_{n=1}^{\infty} \theta^{-n} f_{n}, \quad g(\theta)=\sum_{n=1}^{\infty} \theta^{-n} g_{n}, \quad \theta \rightarrow \infty .
$$

\footnotetext{
1) Исходные асимптотики предполагаются дифференцируемыми.
}

\footnotetext{
* Институт математики Уфимского научного центра РАН, Уфа, Россия. E-mail: klem@imat.rb.ru
} 
Растушие решения удобнее исследовать в переменных амплитуда-фаза, сделав замену $x=r \cos \psi, y=r \sin \psi$. Для функций $r(\theta), \psi(\theta)$ уравнения приобретают вид

$$
\frac{d r}{d \theta}=f \cos \psi+g \sin \psi, \quad\left(\frac{d \psi}{d \theta}+\phi(\theta)-\lambda r^{2}\right) r=g \cos \psi-f \sin \psi .
$$

Основной результат работы состоит в построении асимптотики двухпараметрического семейства решений с неограниченно растущей амплитудой $r(\theta)$. Кроме того, построена асимптотика другого двухпараметрического семейства решений с ограниченной амплитудой.

Структура асимптотик в основном определяется главными коэффициентами в разложении исходных данных и параметром $\lambda$. С одной стороны, для понимания результатов достаточно ограничиться случаем $\varphi(\theta) \equiv \theta, f \equiv 1, g \equiv 0$. С другой стороны, все результаты допускают обобщение на более сложные нелинейные уравнения, которые здесь не обсуждаются. В данной работе анализируется сравнительно частная система со специфической кубической нелинейностью (1). Как раз системы такого типа возникают при асимптотическом решении ряда задач с мальм параметром [1], [2]. Они интенсивно исследуются в последние годы [3], в частности в связи с некоторыми задачами небесной механики. При постановке задачи для системы (1) мы ориентируемся на проблему авторезонанса, которая обычно связывается с физикой плазмы и с ускорителями релятивистских частиц [4]. Большое число статей на эту тему можно найти по адресу http://socrates.berkeley.edu/ fajans/pub.

Решения с растушей амплитудой $r(\theta)$ описывают начальный этап авторезонанса. Именно на исследование авторезонанса нацелены как указанная в (2) специфическая структура коэффициента $\varphi(\theta)$ с ростом на бесконечности, так и постановка вопроса о поиске растуших решений [5]. Вопрос об асимптотике на другой бесконечности (при $\theta \rightarrow$ $-\infty)$ сводится к рассматриваемому случаю $(\theta \rightarrow \infty)$ заменой $(x, y, \theta, \lambda)$ на $-(x, y, \theta, \lambda)$.

Постановка задачи об асимптотике решения в особой точке, в частности на бесконечности, является классической, и на эту тему имеется большое число публикаций (см., например, монографии [6]). Однако для уравнений (1) с данными (2) известные результаты непосредственно не применимы из-за осциллируюшего характера решений. Впрочем, для рассматриваемых уравнений имеется несколько решений с чисто степенной асимптотикой, анализ которых довольно тривиален.

\section{2. СТЕПЕННЫЕ АСИМПТОТИКИ}

В случае, когда входящий в уравнения параметр $\lambda$ положителен, легко построить степенные ряды

$$
r(\theta)=\rho \theta^{1 / 2}+\sum_{k=2}^{\infty} \theta^{-k / 2} \rho_{k}, \quad \psi(\theta)=\psi_{0}+\sum_{k=1}^{\infty} \theta^{-k / 2} \psi_{k}, \quad \theta \rightarrow \infty
$$

которые представляют собой формальное асимптотическое решение при $\theta \rightarrow \infty$. Константы $\rho_{k}, \psi_{k}$ при $k \geqslant 1$ определяются однозначно из рекуррентных формул, получаюшихся при подстановке рядов (4) в уравнения (3). Главные члены растушего решения 
извлекаются из нелинейных уравнений $\left(1-\lambda \rho^{2}\right)=0, \cos \psi_{0}=0$, которые разрешимы при $\lambda>0$. Поскольку тригонометрическое уравнение имеет два различных (по модулю $2 \pi$ ) корня $\psi_{0}= \pm \pi / 2$, то возможно построение двух асимптотических решений. Эти две асимптотические конструкции связаны с разными точными решениями.

ТЕОрема 1. Если $\lambda>0$, то для уравнений (3) с исходными данными (2) существует единственное решение с растущей амплитудой, которое имеет асимптотическое разложсние в виде рядов (4) с $\psi_{0}=\pi / 2$.

ТЕОрема 2. Если $\lambda>0$, то для уравнений (3) с исходными данными (2) существует однопараметрическое семейство решений с растущей амплитудой, которые имеют одно и то же асимптотическое разложение в виде рядов (4) с $\psi_{0}=-\pi / 2$. Разные решения әкспоненциально близки при $\theta \rightarrow \infty$.

Обоснование степенных асимптотик приведено, например, в работе [7].

\section{3. ВКБ-АСИМПТОТИКИ С ОГРАНИЧЕННОЙ АМПЛИТУДОЙ}

В случае $\lambda<0$ не сушествует решений, растущих при $\theta \rightarrow \infty$. Имеет место

ЛЕмма 1. Если $\lambda<0$, то любое решение уравнений (1) с исходными данными (2) ограничено при $\theta \rightarrow \infty$.

Доказательство ограниченности квадрата амплитуды $r^{2}=x^{2}+y^{2}$ вытекает из интегрального тождества

$$
-\lambda r^{4}(\theta)=-\lambda r^{4}(0)-2 \int_{0}^{\theta} \varphi^{\prime}(\zeta) r^{2}(\zeta) d \zeta+4 \int_{0}^{\theta}\left[f^{\prime} y-g^{\prime} x\right] d \zeta
$$

которое является следствием дифференциальных уравнений.

Ограниченные решения сушествуют также и в случае $\lambda>0$. В данном разделе исследуется асимптотика всех ограниченных решений. Структура этих асимптотик не зависит от знака $\lambda$, поэтому дальнейшие построения справедливы как при $\lambda<0$, так и при $\lambda>0$. Однако следует помнить, что при $\lambda<0$ эти асимптотики описывают все решения, тогда как при $\lambda>0$ они описывают лишь множество ограниченных решений. Асимптотика растущих решений будет исследована в следующем разделе.

ТЕОрема 3. Любое ограниченное при $\theta \rightarrow \infty$ решение уравнений (1) с исходными данными (2) разлагается в асимптотический ряд по обратным степеням:

$$
\left(\begin{array}{l}
x \\
y
\end{array}\right)=c\left(\begin{array}{c}
\sin \eta \\
\cos \eta
\end{array}\right)+\sum_{n=1}^{\infty} \theta^{-n} \mathbf{x}_{n}(\eta ; c), \quad \theta \rightarrow \infty
$$

коэффициенть которого $2 \pi$-периодичны по бистрой переменной $\eta=\theta^{2} / 2-\lambda c^{2} \theta+$ $\phi_{1} \ln \theta+s$. Все эти решения могут быть параметризованы двумя константами асимптотики $x=x(\theta ; c, s), y=y(\theta ; c, s) \quad \forall c, s=$ const. 
СлЕДСтвИЕ 1. В частном случае при с $=0$ получается чисто степенная асимптотика единственного решения, стремящегося $к$ нулю на бесконечности:

$$
\left(\begin{array}{l}
x \\
y
\end{array}\right)=-\theta^{-1}\left(\begin{array}{l}
0 \\
1
\end{array}\right)+\sum_{n=2}^{\infty} \theta^{-n} \mathbf{x}_{n, 0}, \quad \theta \rightarrow \infty, \quad \mathbf{x}_{n, 0}=\text { const }
$$

ДОКАЗАТЕЛЬСТВО ТЕОРЕМЫ 3 основано на построении асимптотического решения методом типа ВКБ. При этом нелинейности и правые части в уравнениях (1) рассматриваются как слабые возмушения линейного оператора, так что для определения $\mathbf{x}_{n}$ на каж дом шаге возникает линейная система уравнений. Асимптотическая структура ряда обеспечивается требованием ограниченности коэффициентов $\mathbf{x}_{n}(\eta ; c)$ равномерно по $\eta$.

Вектор-функции $\mathbf{x}_{n}(\eta ; c)=\left(x_{n}, y_{n}\right)^{\mathrm{T}}$ оказываются $2 \pi$-периодическими по $\eta$. Они определяются из неоднородных уравнений линейного осциллятора

$$
\frac{d}{d \eta}\left(\begin{array}{l}
x_{n} \\
y_{n}
\end{array}\right)+\left(\begin{array}{c}
-y_{n} \\
x_{n}
\end{array}\right)=\mathbf{f}_{n}(\eta ; c)
$$

которые получаются из уравнений (1) приравниванием выражений при одинаковых степенях $\theta$. Векторы правых частей выписываются через предыдушие приближения, например

$$
\begin{gathered}
\mathbf{f}_{0} \equiv 0, \quad \mathbf{f}_{1}=\left(\begin{array}{c}
1 \\
0
\end{array}\right)+\lambda\left|\mathbf{x}_{0}\right|^{2}\left(\begin{array}{c}
-y_{0} \\
x_{0}
\end{array}\right)+\lambda c^{2} \mathbf{x}_{0}^{\prime} \\
\mathbf{f}_{2}=\left(\begin{array}{c}
f_{1} \\
g_{1}
\end{array}\right)+\lambda\left|\mathbf{x}_{0}\right|^{2}\left(\begin{array}{c}
-y_{1} \\
x_{1}
\end{array}\right)-\left[\phi_{1}-2 \lambda\left(\mathbf{x}_{0}, \mathbf{x}_{1}\right)\right]\left(\begin{array}{c}
-y_{0} \\
x_{0}
\end{array}\right)+\lambda c^{2} \mathbf{x}_{1}^{\prime}-\phi_{1} \mathbf{x}_{0}^{\prime} .
\end{gathered}
$$

Главньй член асимптотики $\mathbf{x}_{0}(\eta ; c)=c(\sin \eta, \cos \eta)^{\mathrm{T}}$ определяется с точностью до произвольного множителя $c$. Эта константа и сдвиг фазы $s$ представляют два произвольных параметра конструируемого асимптотического решения. На каждом последуюшем шаге решение $\mathbf{x}_{n}(\eta ; c)$ определяется с точностью до двух констант - множителей при паре решений однородной системы. Эти произволы ликвидируются на последующих шагах с помощью требований периодичности поправок по быстрой переменной $\eta$. На первых двух этапах (секулярное) условие ограниченности обеспечивается выбором коэффициентов при $\theta$ и $\ln \theta$ в фазовой функции. Получаемый при этом сдвиг фазы $-\lambda c^{2} \theta$ в быстрой переменной связан с кубическим резонансом, без этого сдвига конструкция асимптотического решения в форме (5) невозможна. Логарифмический сдвиг фазы $\phi_{1} \ln \theta$ возникает благодаря медленно убьваюшему слагаемому в коэффициенте $\varphi(\theta)$, он характерен для линейных уравнений с коэффишиентами такого типа. На этом пути для первой поправки получается выражение

$$
\mathbf{x}_{1}=-\left(\begin{array}{l}
0 \\
1
\end{array}\right)+\left(2 \lambda c-2 \lambda^{2} c^{2} g_{1}-\phi_{2}\right)\left(\begin{array}{c}
\cos \eta \\
-\sin \eta
\end{array}\right) .
$$


Таким образом строится полное формальное асимптотическое решение.

Для обоснования асимптотики следует выделить отрезок построенного ряда (5) $\mathbf{X}_{N}(\theta)$, состояший из первых $N$ слагаемых. Точное решение ишется в виде суммы $\mathbf{x}=$ $\mathbf{X}_{N}(\theta)+\mathbf{Z}_{N}(\theta)$. В задаче для $\mathbf{Z}_{N}$ нетрудно доказать сушествование убываюшего решения $\mathbf{Z}_{N}(\theta)=\mathcal{O}\left(\theta^{-N}\right), \theta \rightarrow \infty$, если воспользоваться линейностью главной части оператора и известными ВКБ-оценками [8] в комбинации с методом сжатых отображений. Таким образом доказывается сушествование решения с заданной асимптотикой (5). Получаемое при этом решение $\mathbf{x}\left(\theta ; c, s_{0}\right)$ гладко зависит от параметров $c, s_{0}$.

Чтобы доказать, что любое ограниченное решение $\mathbf{x}(\theta)$ имеет асимптотику такого типа, надо воспользоваться наличием двух произвольных констант $c, s$ в построенном выше двухпараметрическом семействе решений $\mathbf{x}(\theta ; c, s)$. Эти константы следует определить из требования согласования двух решений $\mathbf{x}\left(\theta_{0}\right)=\mathbf{x}\left(\theta_{0} ; c, s\right)$ в некоторой точке $\theta_{0}$. Отсюда будут следовать их тождественное совпадение $\mathbf{x}(\theta)=\mathbf{x}(\theta ; c, s)$ при любом $\theta$ (в силу единственности) и асимптотика (5) для $\mathbf{x}(\theta)$. Таким образом, доказательство этой части теоремы сводится к нахождению корней $c, s$ системы алгебраических уравнений. Эта задача решается на уровне теоремы сушествования с использованием последовательных приближений. В качестве исходного приближения берутся константы, определяемые из согласования взятого решения с главным членом асимптотики $\mathbf{x}_{0}\left(\eta\left(\theta_{0}, s_{0}\right) ; c_{0}\right)=\mathbf{x}\left(\theta_{0}\right)$. Последуюшие приближения $c_{n}, s_{n}$ находятся из уравнений

$$
\mathbf{x}_{0}\left(\eta\left(\theta_{0}, s_{n}\right) ; c_{n}\right)=\mathbf{x}\left(\theta_{0}\right)+\mathbf{x}_{0}\left(\eta\left(\theta_{0}, s_{n-1}\right) ; c_{n-1}\right)-\mathbf{x}\left(\theta_{0} ; c_{n-1}, s_{n-1}\right) .
$$

Для сдвига фазы берется главное значение $s_{n} \in[0,2 \pi)$. Используя равномерную ограниченность двух рассматриваемых решений, нетрудно получить равномерную по $\theta_{0}$, $n$ ограниченность приближенных амплитуд $c_{n}$. Затем следует воспользоваться гладкостью по параметрам $c, s$ и равномерной оценкой остатка $\mathbf{x}_{0}\left(\eta\left(\theta_{0}, s\right) ; c\right)-\mathbf{x}\left(\theta_{0} ; c\right)=$ $\mathcal{O}\left(\theta_{0}^{-1}\right), \theta_{0} \rightarrow \infty$. Эти свойства обеспечивают сходимость последовательных приближений при достаточно большом значении $\theta_{0}$. Предельные значения и дают искомые корни $c_{n}, s_{n} \rightarrow c, s$. Теорема доказана.

Описанная выше конструкция формального решения соответствует методам ВКБ и двухмасштабных разложений в наиболее простой формулировке. Она оказывается довольно громоздкой. Имеется другой способ, основанный на идеях Крылова-Боголюбова [1], [9], который представляется более эффективным благодаря разбиению всей процедуры на два этапа. Приведем краткое описание этого подхода, который мы будем использовать при анализе растущих решений.

В случае ограниченных решений главный член асимптотики выбирается в виде вектора $\mathbf{x}_{0}=A(\sin S, \cos S)^{\mathrm{T}}$ с неопределенными пока функциями $A, S(\theta)$, для которых выписываются аналоги уравнений эйконала и переноса

$$
\frac{d S}{d \theta}=P(\theta, A), \quad \frac{d A}{d \theta}=Q(\theta, A)
$$


Решение этих уравнений откладывается на второй этап, а на первом этапе строится асимптотика правых частей

$$
P(\theta, A)=\theta+\sum_{n=0}^{\infty} \theta^{-n} P_{n}(A), \quad Q(\theta, A)=\sum_{n=0}^{\infty} \theta^{-n} Q_{n}(A), \quad \theta \rightarrow \infty .
$$

Эти ряды конструируются вместе с асимптотическим решением

$$
\mathbf{x}(\theta)=\sum_{n=0}^{\infty} \theta^{-n} \mathbf{x}_{n}(S ; A), \quad \theta \rightarrow \infty .
$$

Поправки $\mathbf{x}_{n}(S ; A), n>0$, определяются как частные решения неоднородных уравнений линейного осциллятора. При этом коэффишиенты $P_{n}(A), Q_{n}(A)$ рядов $(7)$ входят в правые части уравнений для $\mathbf{x}_{n+1}(S ; A)$ и определяются попарно на каждом шаге из требований периодичности решений по переменной $S$. Две произвольные константы $c, s$ остаются в решениях дифференциальных уравнений (6).

Получаемое таким способом асимптотическое решение является более обшим. Например, первые два члена асимптотики описываются формулами

$$
\begin{gathered}
\left(\begin{array}{l}
x \\
y
\end{array}\right)=A\left(\begin{array}{c}
\sin S \\
\cos S
\end{array}\right)-\theta^{-1}\left(\begin{array}{l}
0 \\
1
\end{array}\right)+\mathcal{O}\left(\theta^{-2}\right), \quad A=c e^{-\lambda g_{1} \theta^{-2}}+\mathcal{O}\left(\theta^{-3}\right), \\
S=\frac{\theta^{2}}{2}-\lambda c^{2} \theta+\phi_{1} \ln \theta+s+\theta^{-1}\left(2 \lambda c-2 \lambda^{2} c^{2} g_{1}-\phi_{2}\right)+\mathcal{O}\left(\theta^{-2}\right), \quad \theta \rightarrow \infty,
\end{gathered}
$$

из которых при переразложении вытекает полученное ранее приближение (5).

\section{4. ВКБ-АСИМПТОТИКИ С НЕОГРАНИЧЕННОЙ АМПЛИТУДОЙ}

В случае $\lambda>0$ помимо ограниченных решений сушествуют растушие. В этом разделе методом ВКБ строится асимптотика двухпараметрического семейства растущих решений. Хотя таких решений оказывается много, остается открытым вопрос о том, исчерпываются ли этим все неограниченные решения.

Теорема 4. Если $\lambda>0$, то уравнения (3) с исходныцми данными (2) имеют двухпараметрическое семейство растущих решений $r\left(\theta ; c, s_{0}\right), \psi\left(\theta ; c, s_{0}\right)$ с асимптотикой

$$
\begin{aligned}
& r(\theta)=\left(\frac{\theta}{\lambda}\right)^{1 / 2}+\theta^{-1 / 4} \sum_{n=1}^{\infty} \theta^{-n / 8} r_{n}(S ; c), \\
& \psi(\theta)=\frac{\pi}{2}+\sum_{n=1}^{\infty} \theta^{-n / 8} \psi_{n}(S ; c), \quad \theta \rightarrow \infty .
\end{aligned}
$$

Коэффичиенты являются $2 \pi$-периодическими функииями по фазовой переменной $S$, которая имеет асимптотику

$$
S=\frac{4 \sqrt{2}}{5} \lambda^{1 / 4} \theta^{5 / 4}+s_{0,1}(c) \ln \theta+\sum_{n=-4}^{\infty} s_{n}(c) \theta^{-n / 4}, \quad \theta \rightarrow \infty .
$$


СлЕДСТВИЕ 2. Первые поправки $к$ главным членам асимптотики описъваются формулами $r_{1}=c \mu^{-1} \sin S, \psi_{1}=-c \cos S, s_{-4}=-\mu c^{2} / 8$.

ДОКАЗАТЕЛЬСТВО ТЕОРЕМЫ 4. Для построения решения с неограниченной амплитудой удобно выделить растущую часть, сделав в уравнениях (3) замену функций

$$
r=\rho \theta^{1 / 2}+\theta^{-1 / 4} \mu^{-1} R(\theta), \quad \psi=\frac{\pi}{2}+\Psi(\theta)
$$

где $\rho=1 / \sqrt{\lambda}, \mu=\sqrt{2} \lambda^{1 / 4}, \lambda>0$. Уравнения для новых неизвестных записываются в форме

$$
\begin{aligned}
R^{\prime}+\theta^{1 / 4} \mu \sin \Psi & =\theta^{1 / 4} \mu[(1-f) \sin \Psi+g \cos \Psi]+\theta^{-1} \frac{R}{4}-\theta^{-1 / 4} \frac{\mu \rho}{2} \\
\Psi^{\prime}-\theta^{1 / 4} \mu R= & -\rho(\phi-\theta) \theta^{1 / 2}+ \\
& +\theta^{-1 / 2}\left\{\frac{R^{2}}{2} \rho-\mu\left(\mu \rho+\theta^{-3 / 4} R\right)^{-1}[g \sin \Psi+f \cos \Psi]\right\} .
\end{aligned}
$$

Основная трудность рассматриваемой задачи состоит в выделении из этих уравнений главной части, которая обеспечивает построение асимптотики, отличной от тривиальных степенных рядов (4). Конечно, можно догадаться, что асимптотика убывающих решений $R, \Psi=o(1), \theta \rightarrow \infty$, будет определяться из линеаризованных в нуле уравнений. В самом деле, асимптотическое решение может быть построено в виде рядов по степеням, кратным $\theta^{-1 / 8}$, с коэффициентами, которые зависят от быстрой переменной $S=(4 / 5) \mu \theta^{5 / 4}+\mathcal{O}(\theta), \theta \rightarrow \infty$, и от пары произвольных констант $c, s_{0}$. Но этот путь ведет к громоздким и запутанным вычислениям.

В данной работе предлагается другой способ, который оказывается более простым в реализации и который не связан с предположением об убывании решений $R, \Psi$. Он представляет собой аналог нелинейного метода ВКБ [9], [10] в комбинации с идеями Крылова-Боголюбова и основан на использовании обшего решения уравнений математического маятника

$$
\frac{d}{d \sigma} R_{0}+\sin \Psi_{0}=0, \quad \frac{d}{d \sigma} \Psi_{0}-R_{0}=0 .
$$

В основу конструкции положено семейство периодических решений уравнений маятника $R_{0}(\sigma ; E), \Psi_{0}(\sigma ; E)$, которое существует в окрестности устойчивой неподвижной точки $(0,0)$. В качестве параметра $E$ удобно использовать значение интеграла энергии $E=R_{0}^{2} / 2-\cos \Psi_{0}+1$. Отметим, что период $T(E)$ и частота $\omega(E)=2 \pi / T(E)$ рассматриваемых решений гладко зависят от энергии. В частности, разложение частоты по малой величине энергии в главных членах имеет вид $\omega(E)=1-E / 8+\mathcal{O}\left(E^{2}\right), E \rightarrow 0$.

Для уравнений (9) строится асимптотическое решение при $\theta \rightarrow \infty$ с использованием $R_{0}(\sigma ; E), \Psi_{0}(\sigma ; E)$ в качестве главного члена ${ }^{2)}$. Основная идея состоит в том, что в

\footnotetext{
${ }^{2)}$ На использование уравнения маятника в похожей задаче об исследовании нелинейного резонанса указывал Чириков [11].
} 
этих функциях допускаются деформации энергии $E=E(\theta)$ и подбирается подходяшим образом быстрая переменная $\sigma$.

Анзац берется в виде асимптотических рядов по степеням, кратным $\theta^{-1 / 4}$ :

$$
R(\theta)=\sum_{n=0}^{\infty} \theta^{-n / 4} R_{n}(\sigma ; E), \quad \Psi(\theta)=\sum_{n=0}^{\infty} \theta^{-n / 4} \Psi_{n}(\sigma ; E),
$$

с коэффишиентами, зависяшими от переменных $\sigma=S(\theta) / \omega(E), E=E(\theta)$. Ограниченность коэффициентов по быстрой переменной $\sigma$ является дополнительным требованием к анзацу.

Фазовая функшия $S(\theta)$ и энергия $E(\theta)$ подлежат определению наряду с коэффициентами $R_{n}, \Psi_{n}$. Для них выписываются аналоги уравнений эйконала и переноса

$$
\frac{d S}{d \theta}=\mu \theta^{1 / 4} \omega(E), \quad \frac{d E}{d \theta}=\mu Q(E, \theta) .
$$

Эти уравнения решаются на втором этапе после определения асимптотики правой части

$$
Q(E, \theta)=\sum_{n=0}^{\infty} \theta^{-n / 4} Q_{n}(E), \quad \theta \rightarrow \infty .
$$

Вводимые таким образом дополнительные неизвестные $Q_{n}(E)$ находятся на первом этапе из требований периодичности по быстрой переменной $\sigma$ асимптотического решения (11).

Рекуррентная система задач для вектор-функций $\mathbf{U}_{n}(\sigma ; E)=\left(R_{n}, \Psi_{n}\right)^{\mathrm{T}}$ получается из уравнений (9) приравниванием выражений при одинаковых степенях $\theta$ и представляет собой линеаризованные уравнения маятника

$$
\frac{d}{d \sigma}\left(\begin{array}{c}
R_{n} \\
\Psi_{n}
\end{array}\right)+\left(\begin{array}{c}
\Psi_{n} \cos \Psi_{0} \\
-R_{n}
\end{array}\right)=\mathbf{F}_{n}(\sigma ; E)
$$

Правые части $\mathbf{F}_{n}$ выписываются через предыдушие приближения. На каждом шаге они содержат очередной неопределенный коэффициент $Q_{n-1}(E)$, который входит множителем при векторе $\mathbf{V}_{0}=\hat{\partial}_{E} \mathbf{U}_{0}(\sigma ; E)$. Фигурирующий в этом выражении оператор $\hat{\partial}_{E}=\left[\partial_{E}-\left(\omega^{\prime} / \omega\right) \sigma \partial_{\sigma}\right]$ иногда называется усеченной производной. Так, на первых шагах имеют место выражения

$$
\begin{gathered}
F_{1}=-Q_{0} \mathbf{V}_{0} \\
F_{2}=-Q_{1} \mathbf{V}_{0}-Q_{0} \hat{\partial}_{E} \mathbf{U}_{1}-\left(\begin{array}{c}
\rho / 2 \\
0
\end{array}\right)+\frac{1}{2} \Psi_{1}^{2} \sin \Psi_{0}\left(\begin{array}{l}
1 \\
0
\end{array}\right) .
\end{gathered}
$$

Решение неоднородной линеаризованной системы вьписывается методом вариации с использованием решений однородной системы

$$
\mathbf{V}_{1}=\partial_{\sigma} \mathbf{U}_{0}(\sigma ; E), \quad \mathbf{V}_{2}=\partial_{E} \mathbf{U}_{0}(\sigma ; E) \equiv \mathbf{V}_{0}+\frac{\omega^{\prime}}{\omega} \sigma \mathbf{V}_{1}
$$


Отметим,что вектор-функции $\mathbf{V}_{0}(\sigma ; E), \mathbf{V}_{1}(\sigma ; E)$ периодичны по $\sigma$, а растуший множитель во втором решении возникает благодаря зависимости частоты $\omega(E)$ от энергии. Поскольку вронскиан $W\left(\mathbf{V}_{2}, \mathbf{V}_{1}\right)=1$, то обшее решение выписьвается через интегралы по быстрой переменной от кососкалярных произведений в виде

$$
\begin{aligned}
\mathbf{U}_{n}= & \mathbf{V}_{0} \int_{0}^{\sigma}\left[\mathbf{V}_{1}, \mathbf{F}_{n}\right] d \eta+ \\
& +\mathbf{V}_{1} \int_{0}^{\sigma}\left(\left[\mathbf{F}_{n}, \mathbf{V}_{0}\right]+\frac{\omega^{\prime}}{\omega} \int_{0}^{\eta}\left[\mathbf{V}_{1}, \mathbf{F}_{n}\right] d \zeta\right) d \eta+C_{n} \mathbf{V}_{2}+D_{n} \mathbf{V}_{1}
\end{aligned}
$$

с произвольными константами $C_{n}, D_{n}$.

Первый этап построения асимптотического решения сводится к решению уравнений (13) в классе функций, периодических по быстрой переменной.

Лемма 2. Рекуррентная система уравнений (13) разрешима в классе периодических по б функиий при подходящем выборе коэффициентов $Q_{n}(E)$.

ДОКАЗАТЕЛЬСТВО сводится к исключению из формулы (14) секулярных слагаемых, растуших квадратично и линейно по $\sigma$. На этом пути получаются выражения для $Q_{n-1}(E)$ и $C_{n}=C_{n}(E)$ через средние значения подынтегральных функций. Константа $D_{n}$ может быть выбрана любой, например нулем. Хотя при разном выборе $D_{n}=D_{n}(E)$ получаются разные ряды, все они асимптотически совпадают (см. [12]).

В частности, вычисления на первых шести шагах приводят к следуюшим результатам: $Q_{0}=Q_{1}=Q_{2}=Q_{3}=0, Q_{4}=-(4 \mu)^{-1}\left\langle R_{0}^{2}\right\rangle, Q_{5}=0$. Угловыми скобками здесь обозначен интеграл, даюший среднее значение. Среди поправок нулевой оказывается только первая: $\mathbf{U}_{1} \equiv 0$.

На втором этапе исследуется уравнение деформации энергии (12).

Лемма 3. Энергия $E(\theta)=E(\theta ; c)$, определяемая как общее решение уравнения (12), зависит от произвольной константы с и имеет асимптотику

$$
E(\theta ; c)=\frac{c^{2}}{2} \theta^{-1 / 4}+\sum_{n=2}^{\infty} E_{n}(c) \theta^{-n / 4}, \quad \theta \rightarrow \infty .
$$

ДОКАЗАТЕЛЬСТво. Главный ненулевой коэффициент в асимптотике правой части $Q(E, \theta)$ указывает на убывание решения $E(\theta)$ к нулю при $\theta \rightarrow \infty$. Далее используем асимптотику по малой энергии решения уравнения маятника

$$
\left(\begin{array}{l}
R_{0} \\
\Psi_{0}
\end{array}\right)=\sqrt{2 E}\left(\begin{array}{c}
\sin \sigma \omega \\
-\cos \sigma \omega
\end{array}\right)[1+\mathcal{O}(E)], \quad E \rightarrow 0,
$$

из которой вытекает асимптотическая формула для среднего значения $\left\langle R_{0}^{2}\right\rangle=E+$ $\mathcal{O}\left(E^{2}\right), E \rightarrow 0$. Тем самым уравнение для энергии в асимптотическом приближении приобретает вид

$$
\frac{d E}{d \theta}=-\frac{1}{4} \theta^{-1}\left[E+\mathcal{O}\left(E^{2}\right)\right]+\mathcal{O}\left(\theta^{-3 / 2}\right) .
$$


Из этого уравнения легко усматривается структура главного члена асимптотики $E(\theta)=$ const $\cdot \theta^{-1 / 4}+\mathcal{O}\left(\theta^{-1 / 2}\right)$ с произвольной константой интегрирования. Полное асимптотическое разложение (15) строится с учетом полных асимптотик для правой части $Q(E, \theta)$ при $\theta \rightarrow \infty$ и для функции $R_{0}(\sigma ; E)$ при $E \rightarrow 0$. Коэффициенты $E_{n}(c)$ вычисляются по рекуррентным формулам. Выбор первого (произвольного) коэффициента в виде $c^{2} / 2$ сделан для удобства записи последующих формул.

Асимптотика по малой энергии для решения уравнений маятника приводит к следуюшему результату.

СлеДСТВИЕ 3. Главный член асимптотического решения уравнений (9) как сложная функция от $(\sigma, \theta)$ имеет асимптотику

$$
\left(\begin{array}{l}
R_{0} \\
\Psi_{0}
\end{array}\right)(\sigma ; E(\theta))=c \theta^{-1 / 8}\left(\begin{array}{c}
\sin \sigma \omega \\
-\cos \sigma \omega
\end{array}\right)\left[1+\mathcal{O}\left(\theta^{-1 / 4}\right)\right], \quad \theta \rightarrow \infty
$$

Из этой формулы становятся понятными происхождение степеней, кратных $\theta^{-1 / 8}$, и обоснованность метода линеаризации для построения асимптотики решений уравнений (9). Отметим, что в асимптотике (16) в качестве быстрой переменной фигурирует фазовая функция $\sigma \omega=S(\theta)$.

В заключение второго этапа следует проинтегрировать уравнение для фазовой функции (12). Для $S(\theta)$, очевидно, получается разложение по степеням, кратным $\theta^{-1 / 4}$; кроме того, возможно появление члена $\ln \theta$.

Наконец, выписываются ряды (8) по степеням, кратным $\theta^{-1 / 8}$, которые получаются из рядов (11) после переразложения коэффициентов с учетом асимптотики для энергии (15). На этом заканчивается построение асимптотического решения.

Поскольку в главном порядке при $\theta \rightarrow \infty$ функции $R, \Psi$ удовлетворяют уравнениям линейного осциллятора, то обоснование асимптотики (16) можно получить способом, указанным в работе [13]. Теорема 4 доказана.

ЗАМЕЧАНИЕ. В частном случае при $c=0$ асимптотическое разложение (8) сильно вырождается и представляется рядом (4) по степеням, кратным $\theta^{-1 / 2}$, с постоянными коэффишиентами и с $\psi_{0}=\pi / 2$. Помимо этого решения сушествует еше однопараметрическое семейство решений с чисто степенной асимптотикой $(8)$ и с $\psi_{0}=-\pi / 2$ (см. теорему 2), которое формально не содержится в описанной выше конструкции. Для включения его в единую конструкцию следует в задаче для $R, \Psi$ выбрать неустойчивую точку равновесия математического маятника $(0,-\pi)$ в качестве главного члена асимптотики.

\section{5. ЗАКЛЮЧЕНИЕ}

Уравнения главного резонанса (1) при $\lambda>0$ имеют богатые семейства как ограниченных, так и растущих решений. В постановке задачи Коши получение того или иного типа решения зависит от выбора начальной точки. Вопрос об аналитическом описании сепаратрисы, которая отделяет начальные данные разного типа, остается открытым. Численные эксперименты свидетельствуют о довольно сложной спиральной структуре сепаратрисы и о сильной перемежаемости областей в окрестности бесконечности. 
Благодарности. Работа выполнена при поддержке РФФИ (грант № 03-01-00716 и грант поддержки ведуших научных школ № 00-15-96038), а также INTAS (грант № 991068).

\section{Список литературы}

[1] Н.Н. Боголюбов, Ю.А. Митропольский. Асимптотические методы в теории нелинейных колебаний. М.: Наука, 1974.

[2] А. Х. Найфә. Методы возмущений. М.: Мир, 1976; Г. М. Заславский, Р. З. Сагдеев. Введение в нелинейную физику. От маятника до турбулентности и хаоса. М.: Наука, 1977.

[3] А. И. Нейштадт. ПММ. 1975. Т. 39. № 4. С. 621; Дифф. уравнения. 1987. Т. 23. № 12. C. 2060; R. Haberman, E. K. Ho. J. Appl. Mech. 1990. V. 62. P. 941; S. G. Glebov, O. M. Kiselev. Russ. J. Math. Phys. 2002. V. 9. № 1. P. 60.

[4] А. А. Коломенский, А. Н. Лебедев. Теория циклических ускорителей. М.: Физматгиз, 1962; M. S. Livingston. High-energy particle accelerators. New York: Interscience, 1954; K. S. Golovanivsky. Phys. Scripta. 1980. V. 22. P. 126; К.С. Голованивский. Физика плазмы. 1985. T. 11. № 3. C. 295; B. Meerson, L. Friedland. Phys. Rev. A. 1990. V. 41. P. 5233; L. Friedland. Phys. Rev. E. 1997. V. 55. P. 1929; 2000. V. 61. № 4. P. 3732.

[5] Л. А. Калякин. Докл. РАН. 2001. Т. 378. № 5. С. 594; L. A. Kalyakin. Russ. J. Math. Phys. 2002. V. 9. № 1. P. 84.

[6] В. В. Козлов, С. Д. Фурта. Асимптотики решений сильно нелинейных систем дифференциальных уравнений. М.: Изд-во МГУ, 1996; А. Д. Брюно. Степенная геометрия в алгебраических и дифференциальных уравнениях. М.: Наука, 1998.

[7] А. Н. Кузнецов. Функц. анализ и его прилож. 1972. Т. 6. № 2. С. 41.

[8] М. В. Федорюк. Асимптотические методы для линейных обыкновенных дифференциальных уравнений. М.: Наука, 1983.

[9] Г. Е. Кузмак. ПММ. 1951. Т. 23. № 3. С. 515.

[10] С. Ю. Доброхотов, В. П. Маслов. Конечнозонные почти периодические решения в ВКБ-приближениях. В сб.: Итоги науки и техники. Сер. Современные проблемы математики. Т. 15. Ред. Р. В. Гамкрелидзе. М.: ВИНИТИ, 1980. С. 3; F. J. Bourland, R. Haberman. SIAM J. Appl. Math. 1988. V. 48. № 3. P. 737.

[11] Б. В. Чириков. ДАН СССР. 1959. Т. 125. № 5. С. 1015.

[12] Л. А. Калякин. Вестн. УГАТУ. Уфа. 2001. № 1(3). С. 40.

[13] М. В. Федорюк. ЖВМиМФ. 1986. Т. 26. № 2. С. 196. 